\title{
PiSCES: Pictures with social context and emotional scenes with norms for emotional valence, intensity, and social engagement
}

\author{
Elizabeth J. Teh ${ }^{1}$ - Melvin J. Yap ${ }^{1} \cdot$ Susan J. Rickard Liow ${ }^{2}$
}

Published online: 25 August 2017

(C) Psychonomic Society, Inc. 2017

\begin{abstract}
Picture databases are commonly used in experimental work on various aspects of emotion processing. However, existing standardized facial databases, typically used to explore emotion recognition, can be augmented with more contextual information for studying emotion and social perception. Moreover, the perception of social engagement, i.e., the degree of interaction or engagement inferred between the people in target pictures, has not been measured. In this paper, we describe the development of a database comprising 203 black-and-white line drawings depicting people within various situational contexts, and normed on perceived emotional valence, intensity, and social engagement, a new construct. Analyses of ratings collected from 62 young adults ( 30 females, 32 males; mean age 22 years) revealed the typical quadratic relationship between valence and intensity, i.e., stimuli that are more emotionally charged, whether positively or negatively valenced, are more intense than emotionallyneutral stimuli. Moreover, the results showed significant linear and quadratic relationships between valence and social engagement ratings, indicating that emotionally-charged social scenes were perceived as more engaging than emotionallyneutral social scenes. This new database will facilitate investigations of how people perceive and interpret social and
\end{abstract}

Electronic supplementary material The online version of this article (doi:10.3758/s13428-017-0947-x) contains supplementary material, which is available to authorized users.

Elizabeth J. Teh

elizabeth.teh.ny@u.nus.edu

1 Department of Psychology, National University of Singapore, 9 Arts Link, Level 2, Block AS4, Singapore 117570, Singapore

2 Department of Otolaryngology, National University of Singapore, Singapore, Singapore emotional information in everyday interactions, and is offered as a resource to experimenters involved in social and/or emotional processing research.

Keywords Picture stimuli $\cdot$ Social cognition $\cdot$ Emotion processing $\cdot$ Valence $\cdot$ Intensity $\cdot$ Socio-emotional processing

\section{Introduction}

Picture databases are a useful resource for experimental studies aimed at investigating emotion processing and social trait judgment in neurotypical and clinical populations. The term "emotion processing" has been used to refer to the processing of emotional experiences within oneself, as well as the interpretation of emotions in others. However, the questions posed by these two strands of research are different and so are the stimuli they require to provide answers. In this paper, we will refer to the first strand as research on "emotional experience," and to the second as research on "emotion perception." We begin by discussing the limitations of existing picture databases for research on emotion and social processing, and then describe the development of a novel database that addresses many of the problems identified.

\section{Emotional experience: Influence on cognition and behavior}

Research on emotional experience focuses on questions about the influence of a person's emotions on his/her behavior or cognitive processes. Researchers in this area often use emotional and non-emotional stimuli from picture, word, or sound databases. For example, there is empirical evidence that emotional stimuli capture attention more quickly than nonemotional stimuli, and that emotional valence affects a 
person's recall and recognition of pictures (see Murphy \& Isaacowitz, 2008, for review).

Some widely used picture databases in this line of research include the International Affective Picture System (IAPS; Lang, Bradley, \& Cuthbert, 1999), the Geneva Affective Picture Database (GAPED; Dan-Glauser \& Scherer, 2011), and the Nencki Affective Picture System (NAPS; Marchewka, Zurawski, Jednorog, \& Grabowska, 2014). These databases contain diverse types of images across the emotional valence spectrum, such as spiders, snakes, scenes involving animal/human rights violations, people's faces, babies, nature scenes, and inanimate objects. Normative data for these affective databases have been collected using dimensional and/or categorical methods.

The dimensional approach involves participants indicating their emotional responses to given pictures using the SelfAssessment Manikin (Bradley \& Lang, 1994) or using negative-to-positive valence-rating scales (Dan-Glauser \& Scherer, 2011; Marchewka et al., 2014). Researchers have generally reported a quadratic relationship between valence and level of arousal for affective pictures (Dan-Glauser \& Scherer, 2011; Olofsson, Nordin, Sequeira, \& Polich, 2008) and words (Warriner, Kuperman, \& Brysbaert, 2013). Specifically, stimuli that are more emotionally charged, whether with positive or negative valence, are more arousing than emotionally-neutral stimuli. Some authors have also reported that correlations of valence and arousal by female raters are significantly higher than those by male raters, particularly for images with human faces or animals (IAPS, Colden et al., 2008; NAPS, Marchewka et al., 2014). In contrast, the categorical approach involves participants selecting emotion response labels (e.g., happiness, anger, fear) to pictures, and/ or rating how much they felt a given emotion when viewing pictures (e.g., $1=$ not at all, to $7=$ very much), thus allowing researchers to categorize pictures according to the emotions they elicit (e.g., IAPS, Mikels et al., 2005; NAPS, Riegel et al., 2016). For the categorical approach, gender effects are reportedly minimal, indicating generally good agreement between male and female participants on emotion labels, except that arousal ratings by females tend to be higher than male participants' ratings for negative pictures, but lower than male participants' ratings for positive pictures (Bradley, Codispoti, Sabatinelli, \& Lang, 2001; Mikels et al., 2005).

Taken together, both types of norms allow experimenters to select stimuli along emotional dimensions, or to select stimuli eliciting specific emotions, in order to explore affective effects on cognition and behavior. However, as the foregoing databases are normed on the emotional experience of the participants themselves, the pictures are not well-suited for answering research questions pertaining to the ability to accurately recognize and report others' emotions. For this, other researchers have developed picture databases with validated emotion labels, which we discuss next.

\section{Emotion perception: Recognizing emotions in other people}

Emotion perception, the second strand of research, focuses on the individual's ability to recognize others' emotions. Participants are usually presented with pictures showing different emotional expressions, and asked to match, label, or describe the emotions depicted. Using this type of paradigm, cross-cultural researchers have found that many emotions are universally recognized, such as happiness, sadness, anger, fear, and disgust (Biehl et al., 1997; Ekman, 1993), whereas clinical researchers have reported challenges with emotion perception in some neuro-atypical populations. For example, compared to typically developing controls, researchers have reported lower emotion recognition accuracy in people with attention deficit hyperactivity disorder (ADHD; Uekermann et al., 2010) and autism spectrum disorders (ASDs; see Uljarevic \& Hamilton, 2013, for a review). Likewise, emotion perception is also impaired in people with psychiatric disorders such as schizophrenia (Kohler, Walker, Martin, Healey, \& Moberg, 2010), anxiety (e.g., Aigner et al., 2007; Montagne et al., 2006) and depression (Kohler, Hoffman, Eastman, Healey, \& Moberg, 2011), as well as acquired conditions like frontotemporal dementia (Mendez, Lauterbach, \& Sampson, 2008) and traumatic brain injury (Bornhofen \& McDonald, 2008).

One of the most widely used standardized picture databases for emotion perception research is the Pictures of Facial Affect (Ekman \& Friesen, 1976), which contains face photographs of actors posing with a range of facial expressions. Other databases include the NimStim Set of Facial Expressions (Tottenham et al., 2009), the Karolinska Directed Emotional Faces (KDEF; Lundqvist, Flykt, \& Öhman, 1998), the Montreal Set of Facial Displays of Emotion (Beaupré, Cheung, \& Hess, 2000), and the Japanese and Caucasian Facial Expressions of Emotion (JACFEE) and Neutral Faces (JACNeuF; Matsumoto \& Ekman, 1988). Typically, normative data for these databases were collected by asking participants to select a label that best describes the emotion portrayed, and to rate the intensity of that emotion (Biehl et al., 1997; Goeleven, De Raedt, Leyman, \& Verschuere, 2008). For the JACFEE database, Biehl et al. (1997) found that females tended to be more accurate than males at recognizing emotions in faces. To date, however, possible gender differences in ratings for emotion perception have not been explored in many databases (e.g., Goeleven et al., 2008; Tottenham et al., 2009).

One major limitation of the existing facial expression databases is that participants are restricted by the emotion labels presented to them by the researcher (e.g., happy, sad, angry, surprised). In turn, investigators using these databases have a limited range of emotion categories available for research. These limitations could be addressed by using dimensional 
rating methods in normative studies to obtain a more sensitive measure of valence (Tottenham et al., 2009). Further, since emotional valence and arousal are closely-related dimensions of emotion (Lang, Greenwald, Bradley, \& Hamm, 1993), it is possible that emotion processing may depend on the degree of intensity perceived in the actor's face. Thus, there is a need for a suitable affective picture database normed on continuous scales of perceived valence and intensity, so that researchers can manipulate both variables when selecting stimuli for their research questions.

A second limitation is that the ability (or inability) to perceive emotions in faces may not fully reflect an individual's ability to evaluate emotions in general. This is because photographs of faces are devoid of situational contexts. It comes as no surprise that researchers using video- and scenario-based picture stimuli have reported that contextual information facilitates emotion decoding (D’Mello \& Graesser, 2010) and social perception (Deuse et al., 2016; Lieberman, 2006). As Levenson (1999) has pointed out, certain emotions, such as jealousy, pride, and embarrassment, need to be understood in the context of interactions with other people or the environment. For these emotions, isolated face images are not likely to be optimal.

For this reason, some researchers have developed other types of experimental materials to study emotion perception, such as pictures and stories of people within a situational context, slide-shows, and video-clips (see Lartseva, Dijkstra, \& Buitelaar, 2015, for a review of experiments on emotional language in autism). However, there are limitations with customized experimental materials. Many of these sets of stimuli are not widely available and are often pilot-tested only with small groups before being used in experiments (e.g., Balconi \& Carrera, 2007; Deuse et al., 2016). Also, the images may vary in visual complexity and the extent of background objects shown, thereby potentially introducing uncontrolled extraneous factors into experiments. Székely and Bates (2000) reported that picture image sizes (in PDF, TIFF, and JPG formats) positively correlated with subjective visual complexity ratings by participants, and higher complexity significantly facilitated picture-naming accuracy for black-andwhite line drawings. Thus, there is a need for a standardized set of context-based affective pictures, normed on dimensions of valence and intensity, to supplement existing facial affect databases for use in emotion perception research. Such a database would also be useful for social perception research, which we will now discuss.

\section{Perception of social information}

Emotion perception is closely related to processing of social information in real-life interactions. As Olsson and Ochsner (2008) argued, understanding of other people's emotions involves an interpretation of their underlying social intentions.
For example, a stimulus action like a punch might be evaluated as an aggressive or playful gesture depending on one's assessment of the puncher's intent. This assessment is based on the contextual information that precedes or follows the provocative action. Dodge, Murphy, and Buchsbaum (1984) found that children's behavioral responses to provocation were determined by their perception of a peer's intention, rather than the peer's actual intention. Crick and Dodge (1994) subsequently suggested that social competence in children develops through learning to detect and interpret current contextual cues using a memory store of social schemas or expected behaviors. This memory store acts as a kind of personal database and presumably it increases with experience. Additionally, social information-processing may involve evaluation of any emotions (in oneself and others) simultaneously present in the situation (Lemerise \& Arsenio, 2000). Hence, the interpretation of other people's intentions and emotions influences how perceivers plan their behavioral responses, or regulate their own emotional responses to the situation (Olsson \& Ochsner, 2008). The apparently spontaneous drive to interpret social information has been attributed to social motivation, which we now describe.

Social motivation construct Baumeister and Leary (1995) proposed that people have a fundamental need for belongingness, a pervasive desire for emotionally-positive social relationships, which drives affective concern for others' welfare. Hence, people devote resources to processing interpersonal interactions in social contexts, which serve as motivations for adapting one's own behavior to the situation (Baumeister \& Leary, 1995; Ryan \& Deci, 2000). Interpersonal relationships have also been found to be a central dimension in how people interpret the causes for events (e.g., doing something because one is married), more so than other attribution dimensions like controllability and locus (Anderson, 1991). Additionally, Sedikides, Olsen, and Reis (1993) showed that people tend to classify incoming information about others in terms of social bonds or relationships, with more priority being given to those with whom one has some sort of connection.

Social motivation theories implicitly assume that people have a natural ability to perceive and process contextual cues for social meaning. On the other hand, it has been argued that this ability may be lacking in some clinical populations, such as people with ASD (Klin, Jones, Schultz, Volkmar, \& Cohen, 2002), or in young children (Crick \& Dodge, 1994) with more limited experience. While the validity of the social motivation construct is not in dispute, several important questions remain with respect to its underlying assumptions. For example, which features in contextual scenes are relevant or influential for evaluation of interpersonal relations, emotions, and causal attributions to support appropriate self-regulation of behaviors? Are there differences in the strength of social bonds in 
different contexts, and what impact might they have on social motivation? Does the valence or intensity of emotions perceived depend on the social context or strength of bonds? Concomitantly, how are valence and intensity evaluated in scenes involving a single person, given the absence of interpersonal relationships? Answers to these questions will extend theories of social motivation, context-based emotion perception, and behavioral self-regulation. To explore these questions empirically, we need a reliable database of picture stimuli. For this reason, we developed the PiSCES set of pictures, which are not only differentiated affectively as in existing databases, but also on the absence or (extent of) presence of social engagement depicted, as described below.

Proposed construct of "social engagement" When people evaluate a given social scene, they typically recognize a range of social information, including social roles or attributed relationships, types of interactions, and actions in particular settings. Potentially, all of these bits of information are important "signposts" for deciding what behavioral responses are acceptable for the situation, although socially-acceptable behaviors may differ from one culture to another. In other words, the degree of "social engagement" within a given social situation influences each person's behavior, and deficits in perceiving this information could give rise to social faux pas or inappropriate behaviors in real life. For example, it may be appropriate to break into song at a party with friends, but the same behavior would be inappropriate at a public library or bus stop with strangers present. We suggest the term "social engagement" for this multidimensional construct representing various situational factors that may be relevant for regulating one's behavioral or emotional responses in real-life contexts.

To our knowledge, social engagement has not been explicitly operationalized and measured in prior research on social processing. We propose that a global rating could be used to measure variability in the extent of social engagement perceived in different scenarios. To determine this rating, people are likely to form and combine multiple social inferences, such as attributed relationships (e.g., parent-child, close friends, strangers, etc.), social identities or roles (e.g., salesperson, customer, driver, etc.), nature of activities or interactions (e.g., a game, a quarrel, a sales transaction, etc.), settings (e.g., public places or private homes), and so on. The social engagement construct may be different or deficient in some individuals, e.g., young children and those with ASD or ADHD, and so a database of stimuli to test it empirically would be useful for future developmental and clinical research.

Existing stimuli for social perception research Social-cognition researchers studying judgments of social traits often use stimuli from facial expression picture databases. For example, Willis and Todorov (2006) found that participants were able to spontaneously infer social traits, like trustworthiness, attractiveness, or aggressiveness, from faces. However, a major limitation of facial stimuli is that they may simultaneously tap emotion and social processing, making it difficult to tease apart the distinct and interactive effects of social and emotional variables on perception (Olsson \& Ochsner, 2008). Another problem is that isolated facial expression stimuli lack contextual information, leading some researchers to develop customized videoclips or other nonstandardized experimental materials to investigate processing of social information, in both neurotypical (e.g., Deuse et al., 2016; Dodge et al., 1984) and clinical (e.g., Orobio de Castro, Merk, Koops, Veerman, \& Bosch, 2005) populations. Clearly, there is a need for standardized, context-based social stimuli to systematically investigate the foregoing issues.

\section{PiSCES database: The present study}

The empirical gap in studying social engagement, and the limitations in existing experimental stimuli for emotion and social perception research, suggest that a new picture database is needed. In this paper, we present a newly-developed set of picture stimuli, the Pictures with Social Context and Emotional Scenes (PiSCES), which has several advantages over existing databases. First, the pictures were designed to vary systematically on emotional valence (positive, negative and neutral), and social engagement. Thus, all the pictures depict one or more person(s) performing an everyday activity within a familiar situational context. We collected normative rating data for perceived emotional valence, intensity, and social engagement, for all pictures. Second, our database includes emotionally-neutral social pictures, which are sparse in existing databases, and can be used as a comparison baseline in emotion studies. Third, we used black-and-white line drawings to minimize issues related to differences in physical properties, such as luminance, color contrast, and composition, which may affect picture processing in affective databases comprising photographs (see review by Olofsson et al., 2008). Our customized pictures have a uniform appearance, and like other databases using black-and-white line drawings (e.g., Snodgrass \& Vanderwart, 1980), PiSCES stimuli include sufficient detail to be consistent with the complexity of real-life people and objects, but they are free from distractions (unrelated or non-essential information) that might influence social and emotional interpretation (e.g., shadows, patterns on clothing, and other embellishments). The artist was asked to adhere to these guidelines in order to enhance goodness-of-depiction (Bates et al., 2003) and familiarity of the concepts depicted (Snodgrass \& Vanderwart, 1980), and to reduce visual complexity of the picture stimuli (Székely \& Bates, 2000). 
PiSCES is a resource database of 203 pictures. All the pictures and item-level norms are freely available to the scientific community in the Supplementary Materials. To our knowledge, this is the first study to obtain continuous ratings of valence and intensity for pictures of people in situational contexts, alongside the degree of social engagement depicted in pictures. Additionally, in this study, we were able to examine the relationships between perceived valence, intensity, and social engagement ratings in the pictures. We present these pictures for researchers' to use for collecting naming or descriptive data when investigating understanding of emotions and social intentions or interactions. Given that PiSCES pictures vary on social and emotional variables, this database could be used to examine dissociations in social-processing and emotion-processing systems. These applications will be discussed in more detail later in this paper.

\section{Method}

Participants A total of 62 undergraduates, comprising 30 females (mean age $=20.3$ years, $S D=1.0$ ) and 32 males (mean age $=23.9$ years, $S D=2.0$ ), were recruited for this study. Eligibility criteria were that participants had normal or corrected-to-normal vision, and their first language was English. Participants earned credit points as part of their psychology courses, or were reimbursed SGD10 for their time. Ethics approval for this study was obtained from the Institutional Review Board, and informed consent was obtained from all participants prior to beginning the experiment. Participants were informed that they were free to withdraw from participation at any time if they felt discomfort during the tasks.

Materials All 203 pictures in the database are line drawings showing at least one person, up to a maximum of four people, performing an activity (e.g., eating, reading, playing, talking, etc.). We sought to develop approximately half of the pictures of a person alone and half of the pictures with two or more persons, to reflect the range of situations and activities that people experience in real-life. To select suitable picture scenarios, which were also varied in emotional valence and extent of social engagement, resources used for speech and language therapy, children's storybooks, and personal conversations were reviewed. Everyday situations that would be easily recognized by adults and children were then selected, and so the written descriptions prepared for the artist covered a variety of solitary, paired or group scenarios involving children and/or adults, in a range of contextual settings such as the home, school/office, playground, bus-stop, restaurant, and a supermarket (see example in Fig. 1).

The pictures were drawn by the artist using computer software (Adobe Photoshop CS5), with a resolution of 2,480 $\times$ 1,748 pixels. Picture visual complexity, indexed by JPG file

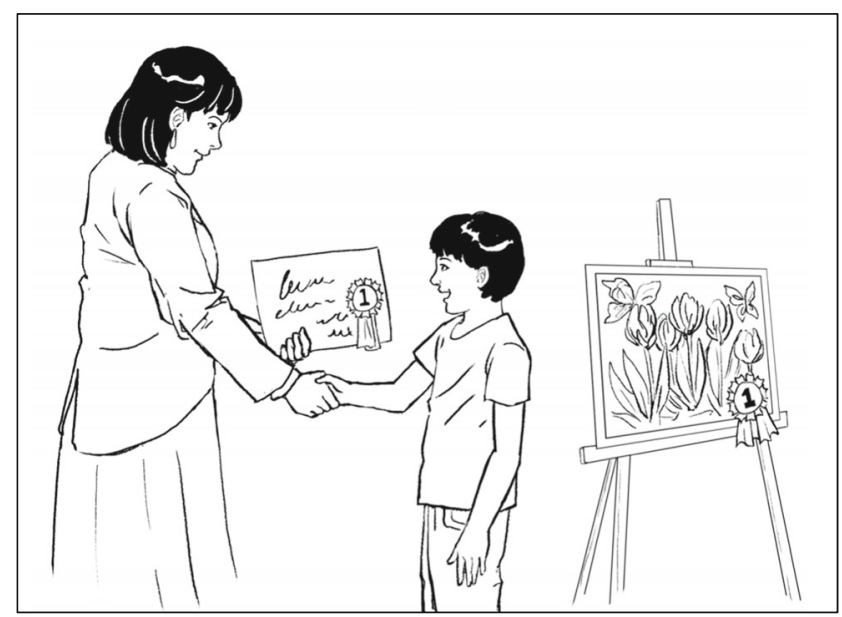

Fig. 1 Example of a picture from the database, depicting people within a contextual situation

sizes (Székely \& Bates, 2000), ranged from $20 \mathrm{kB}$ to $103 \mathrm{kB}$ ( $M=40.18 \mathrm{kB}, S D=11.99 \mathrm{kB}$ ). The main investigator (who is an experienced Speech and Language Therapist), together with the co-authors and a graduate student with previous experience using picture stimuli for child language testing, evaluated all the pictures for quality and clarity of the situations and/or activities depicted. In cases where pictures appeared ambiguous or inadequate for depicting the target scenarios $(n=58$, $28.6 \%$ ), the artist was asked to make amendments, additions and/or deletions to improve the clarity of the pictures. All judges agreed on the pictures for final inclusion in the database.

Procedure Participants were given written information about the experimental tasks and verbally instructed by the experimenter. To collect norms on the emotional conditions represented by the pictures, we asked participants to adopt the perspective of the person(s) in the picture when rating emotional valence and intensity. They practised rating a set of nine example black-and-white pictures that did not overlap with the experimental stimuli, on each of three scale variables. First, participants judged the emotional valence experienced by the person(s) in the picture (from $1=$ strongly negative, $4=$ neutral, to $7=$ strongly positive). Next, participants were asked to rate the intensity of feeling experienced by the person (i.e., perceived arousal; from $1=$ extremely low, unaroused, to $7=$ extremely high, strongly aroused). Finally, we asked participants to judge the degree of social engagement depicted in the picture using the given scale (from $1=$ completely no interaction or engagement with another person, to $7=$ extremely high degree of interaction or engagement with other people).

When participants understood how to perform the task correctly, they were then assigned to a computer, in an individual room with standard lighting. The picture stimuli were presented in randomized order using E-Prime 2.0 software (Psychology Software Tools, Pittsburgh, PA, USA), on a 
desktop computer with a 17 -in. screen. All stimuli were displayed on a white background. Trials began with presentation of a fixation cross $(+)$ displayed in the center of the screen for $500 \mathrm{~ms}$, followed by a blank screen for $500 \mathrm{~ms}$. Then a probe display presented a picture stimulus centered in the upper two-thirds of the screen, and a rating scale centered in the lower one-third of the screen. The stimulus rating scale display terminated when participants indicated their rating for the scale, by pressing a number from 1 to 7 using the number keys on a standard keyboard, and the next rating scale then appeared under the picture. For each stimulus picture, rating scales were presented in a fixed order: emotional valence, intensity, and finally social engagement, to avoid confusing participants as to which scale they were rating. After all three scales had been rated for a picture, a fixation cross appeared again before the next picture was presented with the first of the three rating scales (see Fig. 2). If there was no response on any scale, the next frame appeared after $20 \mathrm{~s}$.

All participants were presented with the complete set of 203 pictures, and they were left alone to complete the task at their own pace, with an untimed (minimum $1 \mathrm{~min}$ ) break slotted in after every 40 pictures. Most of the participants completed the ratings task within $45 \mathrm{~min}$.

\section{Results}

The findings reported below were compiled from 62 participants ( 32 males, 30 females). After collecting data from 62 participants, we separated the data by gender and screened for outliers following a procedure outlined by Schock, Cortese, and Khanna (2012) to eliminate participants who either were not representative of the population of interest or who may not have taken the task seriously. First, each participant's item response rating for every scale was correlated with the overall mean item ratings of all participants of the same gender, for the respective scale. Second, from the set of correlation coefficients obtained, outliers were identified below a cut-off of the first quartile ratings minus 1.5 times the interquartile range of the scale. These cut-off points follow conventions recommended by Tukey (1977). Three outliers $(4.8 \%$, all males) were removed from the dataset, and substituted with three new participants. No further participants fell below the calculated cut-offs on any scale after the replacements. The correlations between each participant's ratings and the overall mean rating of the scale by the same gender were high and significant for all three rating scales: males $M_{\text {valence }}=.89(.05)$, $M_{\text {intensity }}=.74(.10), M_{\text {social }}=.90(.05)$; females $M_{\text {valence }}=$ $.91(.03), M_{\text {intensity }}=.79(.07), M_{\text {social }}=.90(.05)$. Thus, the 62 participants' data were retained for further analyses. All statistical analyses were conducted using SPSS version 24 .

Reliability analyses We conducted reliability analyses on the dataset by calculating two-way random, consistency, averagemeasures intra-class correlation (ICC) of males' and females' ratings for each scale (Hallgren, 2012). ICCs measure the degree of inter-rater agreement, with higher ICCs indicating larger-magnitude agreements (Cicchetti, 1994). The resulting ICCs were in the excellent range on every scale (Table 1), indicating a high degree of agreement among raters within each gender group on the valence, intensity, and social engagement depicted in the set of pictures. Moreover, the high ICCs suggest that measurement error due to rater-effects was minimal, and so statistical power for subsequent analyses was not substantially reduced (Hallgren, 2012). The obtained ratings were therefore deemed suitable for use in the present study.

Gender effects Additionally, we computed item means for ratings by female participants and male participants separately, and correlated them using Pearson's $r$ for each scale. All three scales were highly correlated between males' and females' ratings (emotional valence: $r=.99$; intensity: $r=.98$;

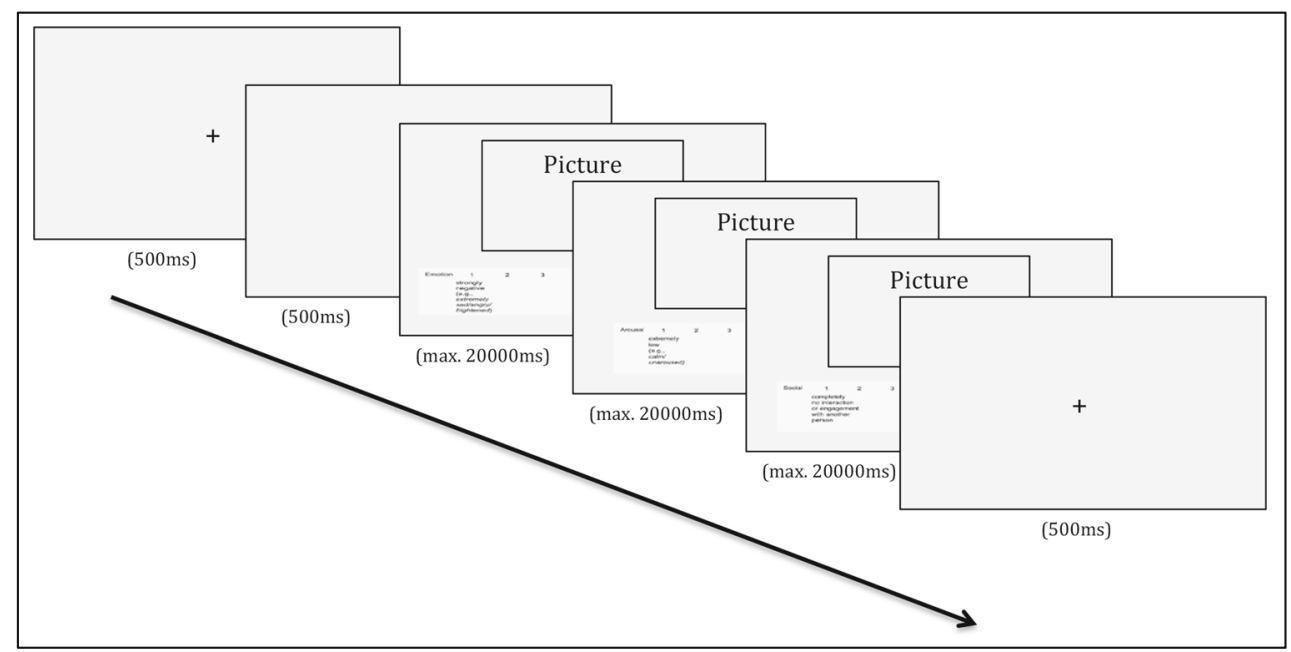

Fig. 2 A graphic overview of the trial design of the rating task 
Table 1 Intra-class correlation coefficients by rating scale and participant gender

\begin{tabular}{llll}
\hline & $\begin{array}{l}\text { Males } \\
(n=32)\end{array}$ & $\begin{array}{l}\text { Females } \\
(n=30)\end{array}$ & $\begin{array}{l}\text { Total } \\
(n=62)\end{array}$ \\
\hline Emotional valence & .99 & .99 & .99 \\
Intensity & .97 & .98 & .99 \\
Social engagement & .99 & .99 & .99 \\
\hline
\end{tabular}

social engagement: $r={ }_{999}$ ). Paired t-tests revealed no significant differences between genders on all three rating scales ( $p$ $>.05$ ). Thus, all further analyses were conducted using the full set of data combined.

Picture means The mean ratings and standard deviations of emotional valence, intensity, and social engagement for each picture, listed in order of overall valence ratings from lowest (i.e., negative valence) to highest (i.e., positive), with valence ratings closer to 4.0 indicating neutral valence, are presented in Appendix 1 in the Supplementary Materials. Descriptive characteristics for the picture database are shown in Table 2 and Fig. 3. For approximately half the pictures, almost all of which depicted only one person, social engagement was rated at, or close to, 1.0 (completely no interaction or engagement with another person). An inspection of picture means revealed a gap between mean ratings up to $1.39(N=103)$ and above $1.90(N=100)$. Therefore, descriptive statistics are presented separately for pictures with social engagement ratings below and above scores of 1.5.

Relationships between emotional valence, intensity and social engagement We aimed to examine how people interpret emotional valence and intensity in others. The relationship between valence and intensity ratings is shown by the scatterplot in Fig. 4a. A hierarchical regression analysis was conducted with mean-centered valence ratings in Block 1, and the squared-values of the mean-centered valence ratings in Block 2. The analysis revealed a significant quadratic relationship between valence and intensity, with the quadratic term accounting for $57 \%$ of the variance in intensity (Table 3a), suggesting that the intensity of emotionally-

Table 2 Descriptive characteristics for the picture database

\begin{tabular}{llll}
\hline $\begin{array}{l}\text { Rating scale } \\
\text { (Scores: } 1 \text { to 7) }\end{array}$ & $N$ & Mean (SD) & Range \\
\hline Emotional valence & 203 & $4.11(1.55)$ & $1.18-6.67$ \\
Intensity & 203 & $4.18(1.45)$ & $1.50-6.69$ \\
Social engagement & 203 & $2.70(1.86)$ & $1.00-6.45$ \\
$\quad$ Pictures rated $\leq 1.5$ & 103 & $1.06(0.08)$ & $1.00-1.39$ \\
$\quad$ Pictures rated $>1.5$ & 100 & $4.39(1.18)$ & $1.90-6.45$ \\
\hline
\end{tabular}

charged pictures was greater than that for emotionallyneutral pictures.

Next, we examined how emotional valence relates to social engagement. As discussed earlier, because the variance on this variable was restricted to 103 pictures, it might not be possible to detect a significant relationship with valence using the full set of pictures. Therefore, we restricted our analyses to the subset of 100 pictures that were rated above 1.5 on social engagement, most of which depicted more than one person. There were significant linear and quadratic effects of emotional valence on social engagement (Table $3 b$ ). Thus, valence positively predicted the degree of social engagement in the pictures, and the effect appeared greater for positive than for negative pictures, but was lower for emotionally-neutral pictures (Fig. 4b). Overall, emotional valence accounted for nearly $43 \%$ of the total variance in social engagement (Table $3 b$ ).

We also examined the relationship between social engagement and intensity in the subset of pictures rated above 1.50 on social engagement. Here, mean-centered social engagement ratings were entered in Block 1, and the squaredvalues of the mean-centered social engagement ratings were entered in Block 2. There was a large positive linear effect $\left(R^{2}\right.$ $=.26$; Table $3 \mathrm{c}$ ), indicating that the greater the degree of social engagement depicted between two or more people, the greater the intensity appeared to raters. Additionally, there was a smaller, significant quadratic effect $\left(\Delta R^{2}=.08\right.$; Table 3c), suggesting that the intensity of some pictures was lower than others. Therefore, we extracted pictures that fell below the midpoint of the intensity scale $(<4.0)$ for further examination. We found that these pictures were also rated closer to neutral on valence, $M=4.01(0.60)$, and social engagement was moderate, $M=3.59(0.80)$. Hence the quadratic curve between intensity and social engagement can be explained by the strongly quadratic relationship between intensity and valence described earlier.

High variability of emotional valence in some 'neutral' pictures On closer inspection of the emotional valence ratings, we noted that a subset of six pictures in the database (3.0\%) had mean ratings between 3.5 and 4.5 (i.e., close to emotionally-neutral), but there was high variation in the ratings given by participants $(S D>1.0$; Table 4$)$. An inspection of the raw data revealed that these images received ratings ranging from 1.0 or 2.0 , to 6.0 or 7.0 , which resulted in valence means close to the mid-point of 4.0 (Table 4).

In other words, participants appeared divided in perceiving these six pictures as either more negative or more positive. (Pictures with mean ratings below 3.5 or above 4.5 were not susceptible to this problem as $S D$ s $>1.0$ remained within the negative or positive ends of the rating scale.) One reason could be that certain scenes are inherently multi-valenced, thus allowing a broader range of acceptable emotional judgments because raters prioritize 
(a)

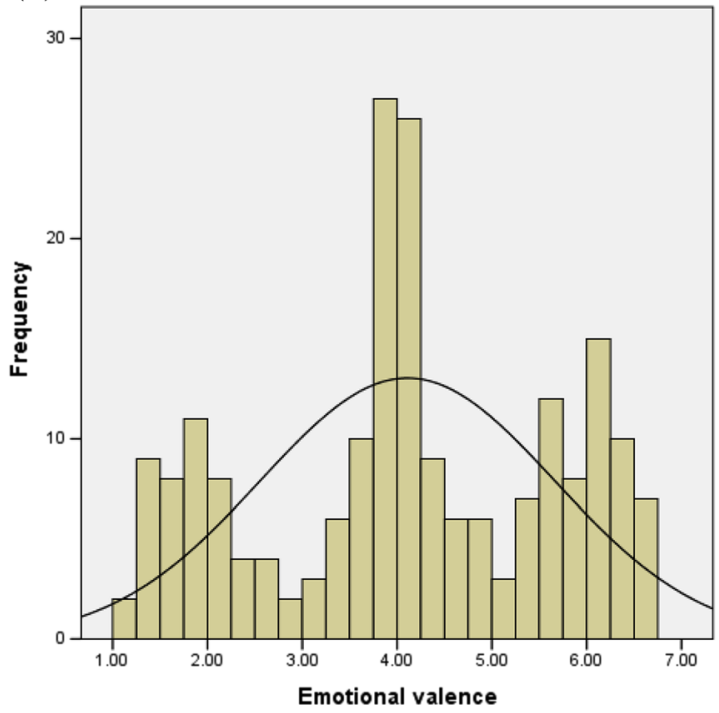

(c)

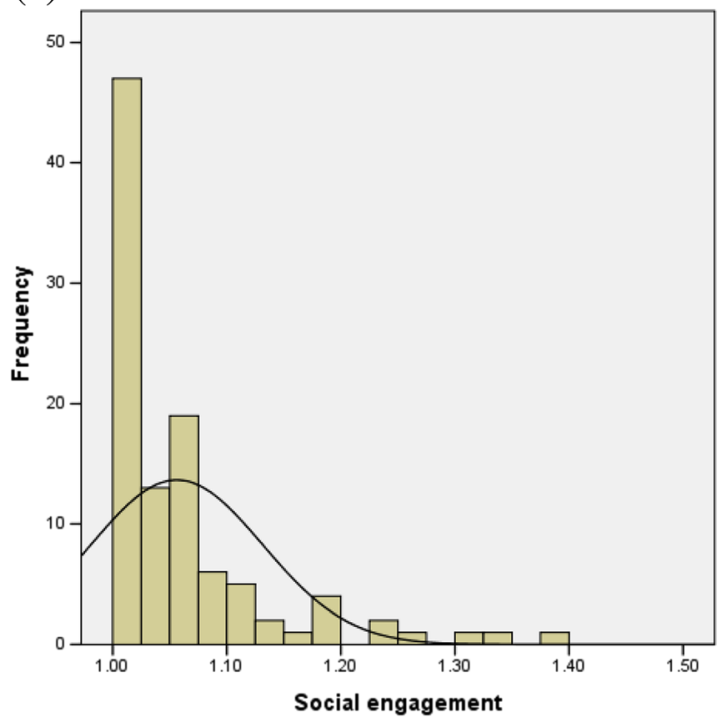

(b)
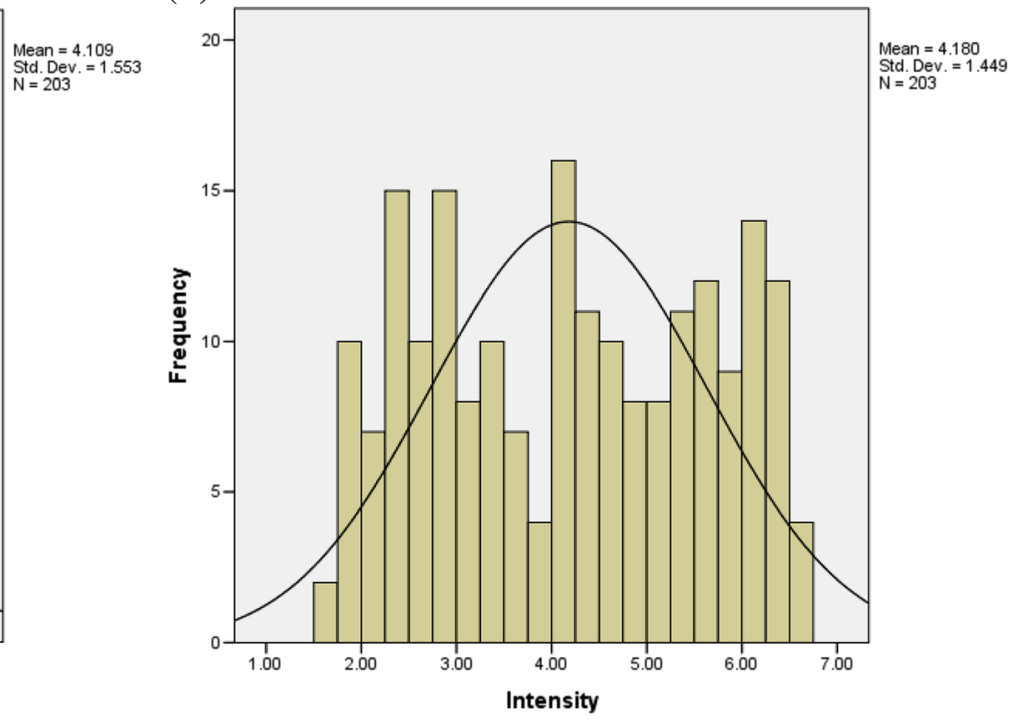

(d)

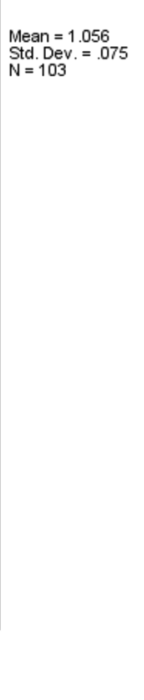

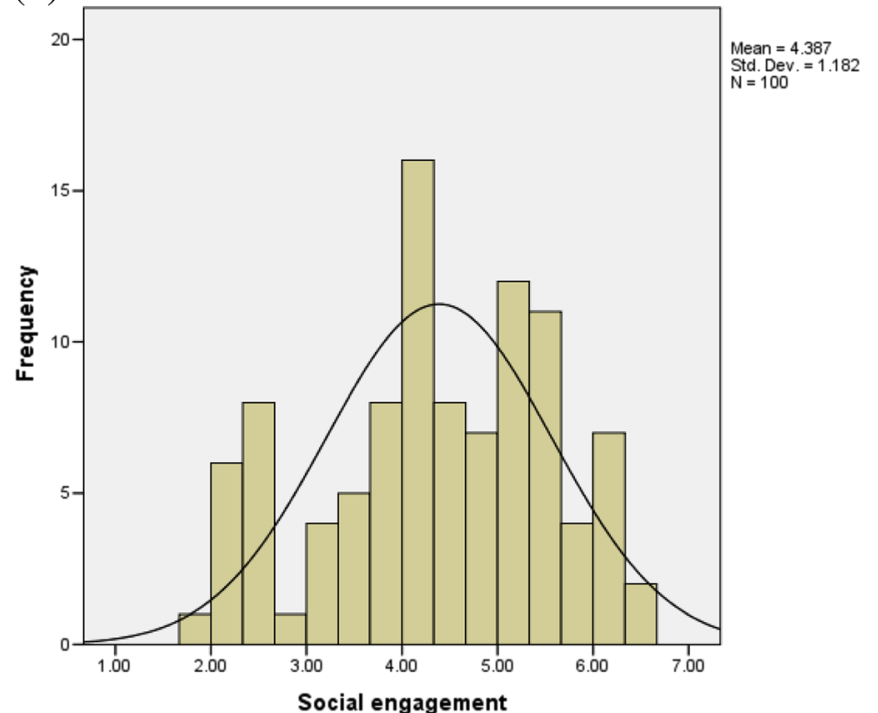

Fig. 3 Distribution of picture ratings for (a) emotional valence, (b) intensity, (c) social engagement of pictures rated $\leq 1.5$, and (d) social engagement of pictures rated $>1.5$

particular individuals in a scene (e.g., a mother comforting her crying baby). Another possible reason is that certain scenes are inherently more ambiguous emotionally (e.g., a "tug-of-war" could be viewed as competitive or fun by different people), because people may construe real-life situations differently depending on factors such as their current goals or prior experiences (Crick \& Dodge, 1994). The variability in this small subset of pictures is comparable to findings reported in other emotion-categorization studies that some images can depict more than one discrete emotion (Mikels et al., 2005; Riegel et al., 2016). For these reasons, we offer this subset as a small group of multivalenced emotional stimuli for researchers, highlighting that the near-neutral valence ratings of these six pictures should be interpreted with caution by future researchers.

\section{Discussion}

In this paper, we have described and shared a new methodological resource for studying social processing and emotional processing involving Pictures with Social Context and Emotional Scenes (PiSCES). The database contains a set of 203 line drawings depicting people in everyday situations that are systematically varied on emotional and social components. More specifically, we ascertained that our pictures represent a range of valence, intensity and social engagement, by obtaining normative ratings using 7-point scales for these three variables. To the best of our knowledge, this is the first study to collect norms on perceived extent of social engagement depicted in pictures. The results showed excellent interrater reliability, suggesting that there was high agreement on 
(a)

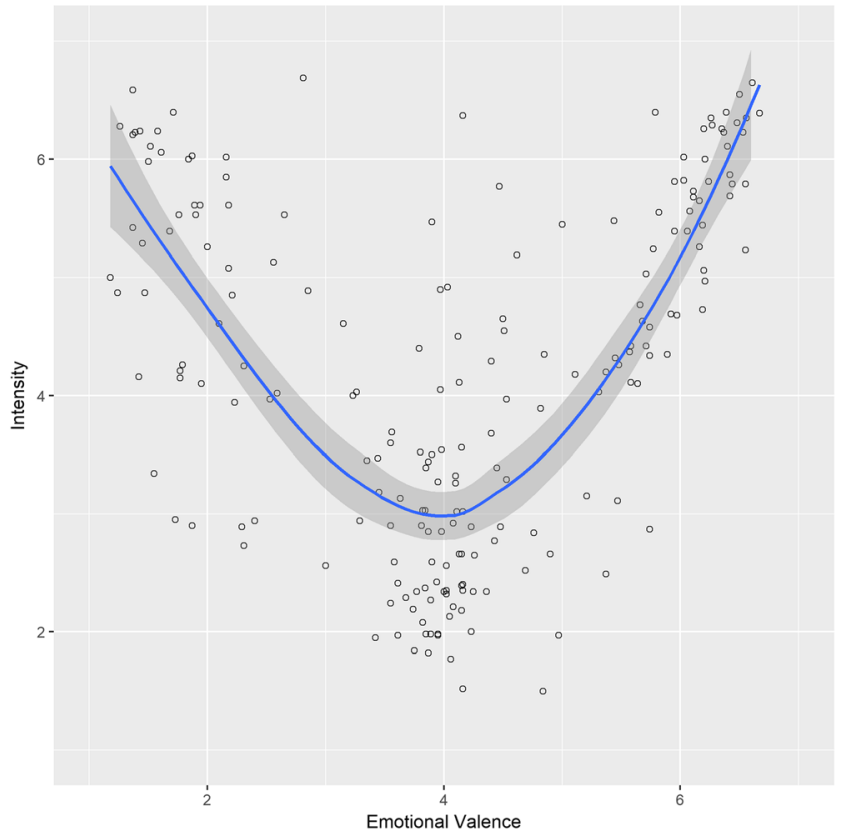

(c)

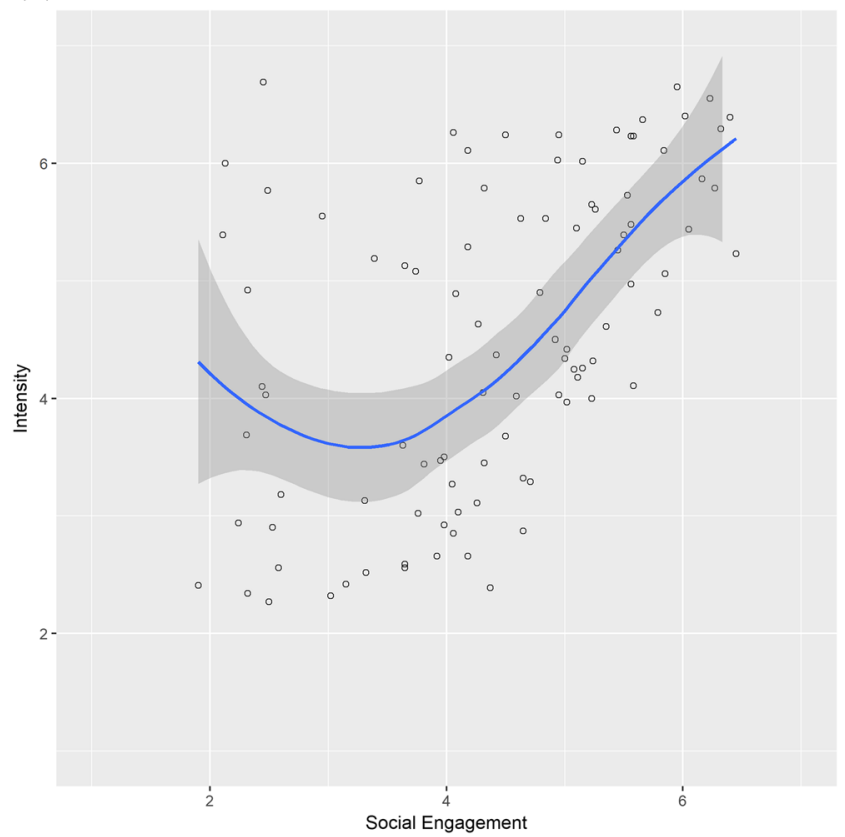

(b)

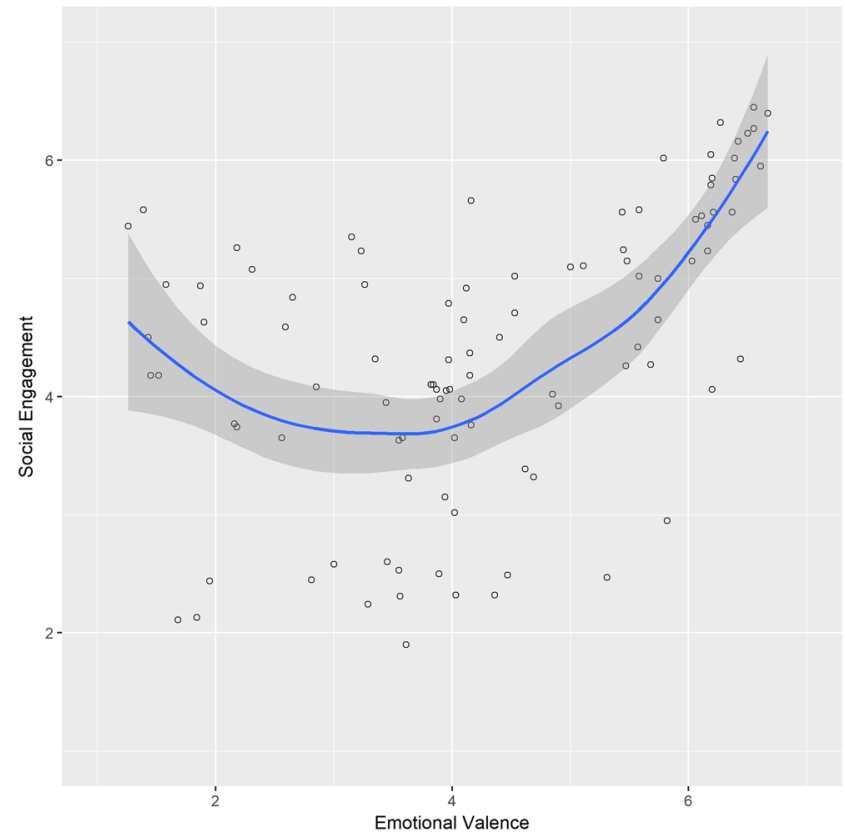

Fig. 4 Scatterplot matrices of (a) emotional valence with intensity $(N=203),(\mathbf{b})$ emotional valence and social engagement $(N=100)$, and (c) intensity and social engagement $(N=100)$

the target concepts underlying the pictures. Moreover, and in line with previous studies reporting minimal gender differences in categorization or labeling of emotions in pictures (Bradley et al., 2001; Mikels et al., 2005), there was very high agreement between males and females on ratings of emotional valence and intensity, as well as social engagement, for our pictures. Finally, we examined relationships between perceived emotional valence and intensity, and social engagement. The main findings of our study are discussed next, before we propose potential uses and future directions for the PiSCES database.

Relationship between emotional valence and intensity The quadratic relationship found between the level of intensity and emotional valence of the pictures indicates that characters perceived as being emotionally-valenced, whether positively or negatively, were also perceived as being more intense than emotionally-neutral characters. This relationship has not been 
Table 3 Summary of hierarchical regressions with (a) emotional valence as a predictor of intensity, (b) valence as a predictor of social engagement, and (c) social engagement as a predictor of intensity

\section{$\beta \quad t \quad p$}

(a) Dependent variable: Intensity

(including all pictures, $N=203$ )

Block 1

Emotional valence (mean-centered)

$.11 \quad 1.62 \quad .107$

$$
F(1,201)=2.62, R^{2}=.01
$$

Block 2

Emotional valence-squared (mean-centered) $\quad .77 \quad 16.60<.001$

$$
F(2,200)=140.79 * * *, \Delta R^{2}=.57
$$

(b) Dependent variable: Social engagement

(pictures with more than one person only, $N=100$ )

Block 1

Emotional valence (mean-centered)

$.47 \quad 5.27<.001$

$$
F(1,98)=27.79 * * *, R^{2}=.22
$$

Block 2

Emotional valence-squared (mean-centered)

$.45 \quad 5.83<.001$

$$
F(2,97)=35.57 * * *, \Delta R^{2}=.20
$$

(c) Dependent variable: Intensity

(pictures with more than one person only, $N=100$ )

Block 1

Social engagement (mean-centered)

$\begin{array}{lll}51 & 5.84<.001\end{array}$

$$
F(1,98)=34.18^{* * *}, R^{2}=.26
$$

Block 2

Social engagement-squared (mean-centered)

$$
F(2,97)=25.21 * * *, \Delta R^{2}=.08
$$

$* * * p<.001$

investigated before with the facial expression databases used for emotion-recognition studies. Thus, this study is the first to demonstrate that the classic "U-shaped" relationship between experienced valence and arousal, which has been reported when measuring raters' emotional responses to given word or picture stimuli (e.g., Olofsson et al., 2008; Warriner et al., 2013), also exists when people evaluate others' emotions. The relationship holds for pictures of single people and pictures of multiple people. Therefore, researchers should be mindful of intensity levels when selecting stimuli for emotionperception studies.
Variability in social engagement construct Our findings for social engagement revealed that people-based picture stimuli vary in the extent of social information presented. In particular, pictures with two or more people ranged in mean ratings from 1.9 to 7.0. Higher social engagement ratings suggested that participants perceived a greater degree of interaction in the scene than in pictures with lower ratings. Our findings support and extend social motivation theories on the centrality of "interpersonalness" when evaluating situations (Anderson, 1991), by showing that there are varying degrees of engagement between people in different contexts. For example, in interactions between two people, interactions between peers (e.g., two girls talking on the phone) were rated more highly in terms of social engagement than interactions with a salesperson or other professional (e.g., a cashier or doctor). Evidently, our participants evaluated social engagement based on features such as the nature of the perceived relationship and interaction, setting, roles, and actions involved. Thus, it is likely that social engagement is a multidimensional construct that taps many features of a social situation. This finding is also consistent with Sedikides et al.'s (1993) observation that information about people was spontaneously used to organize the target stimuli into relationship categories (e.g., married couples or friends). We suggest that these features represent social information relevant for modifying people's behavior (Ryan \& Deci, 2000). That is, it seems plausible that people spontaneously consider, and are influenced by, context-based relationships, roles, actions, and settings when making decisions for behavioral self-regulation.

Relationship between social engagement, valence, and intensity For pictures with two or more people, we found a significant linear and quadratic relationship between emotional valence and social engagement. Emotionally-charged interactions between people, whether negatively-valenced (e.g., two people arguing) or positively-valenced (e.g., two children hugging) tended to be rated higher on social engagement than emotionally-neutral interactions (e.g., someone talking to a

\begin{tabular}{|c|c|c|c|c|c|c|}
\hline Picture & Brief description & $n$ & M & SD & Min & Max \\
\hline Picture 86 & Girl sees chickens outside her house & 62 & 3.90 & 1.28 & 1 & 6 \\
\hline Picture 91 & Woman carrying a crying baby & 61 & 3.97 & 1.53 & 1 & 7 \\
\hline Picture 92 & Children looking into a box & 62 & 3.97 & 1.04 & 2 & 7 \\
\hline Picture 99 & Boy watching a girl on her skateboard & 60 & 4.03 & 1.50 & 1 & 7 \\
\hline Picture 107 & Woman holding a torn bag; boy picking up some items on the floor & 60 & 4.12 & 1.34 & 2 & 7 \\
\hline Picture 117 & Children participating in a "tug-of-war" & 62 & 4.16 & 1.50 & 1 & 7 \\
\hline
\end{tabular}
salesperson). Additionally, this pattern was reflected in the quadratic relationship between intensity and social engagement. Interactions that were greater in social engagement were also greater in intensity. (However, interactions that were

Table 4 Subset of pictures with wide variability in emotional valence ratings 
emotionally-neutral were generally lower in both intensity and social engagement.).Hence, it appears that, when two or more people are present, people tend to attribute greater emotionality (and corresponding intensity levels) to situations with more perceived social engagement. Baumeister and Leary (1995) argued that in interactions, the degree of intimacy between oneself and the other person(s) influences the intensity of emotions experienced. Our results indicate that the same relationship holds for perceived emotionality and social engagement when evaluating other people in presented situations. This relationship may be attributed to a fundamental human need for belongingness (Baumeister \& Leary, 1995). Further, positively-valenced pictures were rated higher on social engagement than negatively-valenced pictures, which may reflect Baumeister and Leary's (1995) argument that people are driven to promote positive relationships in social situations.

These two findings about the relationship between social engagement and emotional valence, as well as social engagement and intensity, both seem worthy of future research. They also serve to caution researchers about the potential impact of perceived social engagement in pictures when investigating emotion processing. Hence, to the extent that cut-offs may be set for valence and social engagement ratings, we propose grouping the pictures into six social/valence conditions (Table 5, and Appendix 2 in the Supplementary Materials).

Potential applications of the PiSCES database Here we draw attention to three main applications of the PiSCES database. First, as the pictures in the PiSCES database are normed on social and emotional variables, they will be useful as experimental stimuli in future emotion and social perception studies. Given that all 203 pictures provide contextual cues to support social and emotional perception, the database should prove more useful than the decontextualized facial expressions currently used in emotion and social trait judgment studies at present. Second, because all the pictures contain people, future researchers will now be able to control for varying levels of social information

Table 5 Grouping of pictures on social and valence dimensions

\begin{tabular}{llll}
\hline Condition & $\begin{array}{l}\text { Social engagement } \\
\text { ratings }\end{array}$ & $\begin{array}{l}\text { Valence } \\
\text { ratings }\end{array}$ & $\begin{array}{l}\text { No. of } \\
\text { pictures }\end{array}$ \\
\hline (1) Low social/negative & 1.0 to $\leq 1.5$ & 1.0 to $<3.5$ & 29 \\
(2) Low social/neutral & 1.0 to $\leq 1.5$ & 3.5 to $\leq 4.5$ & 42 \\
(3) Low social/positive & 1.0 to $\leq 1.5$ & 4.5 to 7.0 & 32 \\
(4) High social/negative & $>1.5$ to 7.0 & 1.0 to $<3.5$ & 28 \\
(5) High social/neutral & $>1.5$ to 7.0 & 3.5 to $\leq 4.5$ & 30 \\
(6) High social/positive & $>1.5$ to 7.0 & 4.5 to 7.0 & 42 \\
\hline
\end{tabular}

in stimuli by referring to the social engagement norms. Similarly, the PiSCES database will enable researchers to tease apart the effects of emotional valence and social variables on processing. Olsson and Ochsner (2008) suggested that a dissociation of emotional and social information was theoretically possible but they noted that the two variables often overlap in experimental stimuli, so that tasks often tap both simultaneously. To illustrate, in neuroscience research, Ochsner (2008) noted that social cognitive studies usually involve evaluating affective processes (like emotions and attitudes), while affective tasks often use social stimuli (like faces). The PiSCES database will enable researchers to select stimuli that are orthogonally differentiated on social and emotional variables, as proposed in Table 5 above, and thereby examine the theoretical dissociability of social and emotional information in pictures.

Third, in developmental research, picture-naming tasks are often used for studying vocabulary acquisition, wordretrieval skills, and semantic representation of nouns and verbs in children from as young as 3 years old (Masterson, Druks, \& Gallienne, 2008). We propose that the PiSCES pictures could be used for similar picturenaming or description tasks, to investigate learning of emotional and social concepts in children. For this purpose, the PiSCES picture database should be normed on children at different ages in future work. Norms would also facilitate research on social cognition and emotionperception processes in clinical populations across the age range. Such research will enhance our understanding of social and emotional processing in developmental and/or clinical populations, and a parallel set of pictures could be developed as a training resource for use during clinical intervention programs.

\section{Limitations and future directions}

Our social engagement scale was a global measure encompassing multiple facets that may influence interactions, like social roles, relationships, actions, intentions, and situational settings, as it was not logical to rate these aspects separately. The ratings were all collected on young undergraduates so it is not yet clear whether the results will be generalizable to people from other age ranges and educational backgrounds, or different ethnic groups. Further work using a naming paradigm, or another measure, will be needed to obtain norms for individual aspects of the social engagement construct. Future researchers may then also investigate how different key features of social contexts are associated with emotional valence.

Finally, because existing picture databases standardized on elicited emotional responses tend to suffer from a lack of emotionally-neutral pictures with people (Colden et al., 2008), our database of emotional and neutral people-based 
scenes could potentially be a useful addition to the resources available for studying effects of emotional valence on cognitive and behavior processes. Hence, while we were more interested in measuring emotional perception than emotional experiences in this study, we suggest that other researchers obtain normative emotional-response ratings for the pictures in the future. In the same way, we leave it open to researchers to collect other norms besides social and emotional ratings in order to use the database in other fields of study.

\section{Conclusion}

In this paper, we introduced a new picture database comprising 203 contextualized scenes of one or more people in everyday situations. All pictures are normed on emotional valence, intensity, and social engagement, a new construct measuring the degree of engagement between people, as perceived by participants. The PiSCES supplements existing databases of facial expressions for emotion recognition studies, and serves as an alternative to other affective databases normed on participants' emotional responses to images. We offer these pictures to researchers as a resource for studying social and emotional processing, and recommend that researchers consider the inter-relationships between valence, intensity and social engagement when selecting social stimuli in future studies.

Acknowledgements We would like to thank Ms Chu Lee Yeang, who drew all the pictures in this database; Ms Choo Rui Qi, who provided valuable inputs on the drafts of the illustrations; and Mr Aaron Lim, our research assistant.

This study was supported by Research Grant R-581-000-176-101 to MY, and the Master of Science (Speech \& Language Pathology) Program, Yong Loo Lin School of Medicine, National University of Singapore.

\section{References}

Aigner, M., Sachs, G., Bruckmuller, E., Winklbaur, B., Zitterl, W., Kryspin-Exner, I., ... \& Katschnig, H. (2007). Cognitive and emotion recognition deficits in obsessive-compulsive disorder. Psychiatry Research, 149, 121-128.

Anderson, C. A. (1991). How people think about causes: Examination of the typical phenomenal organization of attributions for success and failures. Social Cognition, 9, 295-329.

Balconi, M., \& Carrera, A. (2007). Emotional representation in facial expression and script: A comparison between normal and autistic children. Research in Developmental Disabilities, 28, 409-422.

Bates, E., D'Amico, S., Jacobsen, T., Székely, A., Andonova, E., Devescovi, A., ... \& Tzeng, O. (2003). Timed picture naming in seven languages. Psychonomic Bulletin \& Review, 10, 344-380.

Baumeister, R. F., \& Leary, M. R. (1995). The need to belong: Desire for interpersonal attachments as a fundamental human motivation. Psychological Bulletin, 117, 497-529.
Beaupré, M. G., Cheung, N., \& Hess, U. (2000). The Montreal Set of Facial Displays of Emotion. Montreal: Department of Psychology, University of Quebec.

Biehl, M., Matsumoto, D., Ekman, P., Hearn, V., Heider, K., Kudoh, T., \& Ton, V. (1997). Matsumoto and Ekman's Japanese and Caucasian facial expressions of emotion (JACFEE): Reliability data and crossnational differences. Journal of Nonverbal Behavior, 21, 3-21.

Bornhofen, C., \& McDonald, S. (2008). Emotion perception deficits following traumatic brain injury: A review of the evidence and rationale for intervention. Journal of the International Neuropsychological Society, 14, 511-525.

Bradley, M. M., Codispoti, M., Sabatinelli, D., \& Lang, P. J. (2001). Emotion and motivation II: Sex differences in picture processing. Emotion, 1, 300-319.

Bradley, M. M., \& Lang, P. J. (1994). Measuring emotion: The selfassessment manikin and the semantic differential. Journal of Behavioral Therapy and Experimental Psychiatry, 25, 49-59.

Cicchetti, D. V. (1994). Guidelines, criteria, and rules of thumb for evaluating normed and standardized assessment instruments in psychology. Psychological Assessment, 6, 284-290.

Colden, A., Bruder, M., \& Manstead, A. S. R. (2008). Human content in affect-inducing stimuli: A secondary analysis of the international affective picture system. Motivation and Emotion, 32, 260-269.

Crick, N. R., \& Dodge, K. A. (1994). A review and reformulation of social information-processing mechanisms in children's social adjustment. Psychological Bulletin, 115, 74-101.

D’Mello, S. K., \& Graesser, A. (2010). Multimodal semi-automated affect detection from conversational cues, gross body language, and facial features. User Modeling and User-Adapted Interaction, 20, 147-187.

Dan-Glauser, E. S., \& Scherer, K. R. (2011). The Geneva affective picture database (GAPED): A new 730-picture database focusing on valence and normative significance. Behavior Research Methods, 43, 468-477.

Deuse, L., Rademacher, L. M., Winkler, L., Schultz, R., Grunder, G., \& Lammertz, S. E. (2016). Neural correlates of naturalistic social cognition: Brain-behavior relationships in healthy adults. Social Cognitive and Affective Neuroscience, 11, 1741-1751.

Dodge, K. A., Murphy, R. R., \& Buchsbaum, K. (1984). The assessment of intention-cue detection skills in children: Implications for developmental psychopathology. Child Development, 55, 163-173.

Ekman, P. (1993). Facial expression and emotion. American Psychologist, 48, 384-392.

Ekman, P., \& Friesen, W. V. (1976). Pictures of Facial Affect. Palo Alto: Consulting Psychologists Press.

Goeleven, E., De Raedt, R., Leyman, L., \& Verschuere, B. (2008). The Karolinska Directed Emotional Faces: A validation study. Cognition and Emotion, 22, 1094-1118.

Hallgren, K. A. (2012). Computing inter-rater reliability for observational data: An overview and tutorial. Tutorials in Quantitative Methods for Psychology, 8, 23-34.

Klin, A., Jones, W., Schultz, R., Volkmar, F., \& Cohen, D. (2002). Visual fixation patterns during viewing of naturalistic social situations as predictors of social competence in individuals with autism. Archives of General Psychiatry, 59, 809-816. 
Kohler, C. G., Hoffman, L. J., Eastman, L. B., Healey, K., \& Moberg, P. J. (2011). Facial emotion perception in depression and bipolar disorder: A quantitative review. Psychiatry Research, 188, 303-309.

Kohler, C. G., Walker, J. B., Martin, E. A., Healey, K. M., \& Moberg, P. J. (2010). Facial emotion perception in schizophrenia: A meta-analytic review. Schizophrenia Bulletin, 36, 1009-1019.

Lang, P. J., Bradley, M. M., \& Cuthbert, B. N. (1999). International Affective Picture System (IAPS): Instruction manual and affective ratings. (Tech. Rep. No. A-4). Gainsville: University of Florida, Center for Research in Psychophysiology.

Lang, P. J., Greenwald, M. K., Bradley, M. M., \& Hamm, A. O. (1993). Looking at pictures: Affective, facial, visceral, and behavioral reactions. Psychophysiology, 30, 261-273.

Lartseva, A., Dijkstra, T., \& Buitelaar, J. K. (2015). Emotional language processing in autism spectrum disorders: A systematic review. Frontiers in Human Neuroscience, 8, 991.

Lemerise, E. A., \& Arsenio, W. F. (2000). An integrated model of emotion processes and cognition in social information processing. Child Development, 71, 107-118.

Levenson, R. W. (1999). The intrapersonal functions of emotion. Cognition and Emotion, 13, 481-504.

Lieberman, M. D. (2006). Neural bases of situational context effects on social perception. Social Cognitive and Affective Neuroscience, 1, 73-74.

Lundqvist, D., Flykt, A., \& Öhman, A. (1998). The Karolinska Directed Emotional Faces - KDEF, CD ROM from Department of Clinical Neuroscience, Psychology section, Karolinska Institutet, ISBN 91630-7164-9.

Marchewka, A., Zurawski, L., Jednorog, K., \& Grabowska, A. (2014). The Nencki Affective Picture System (NAPS): Introduction to a novel, standardized, wide-range, high-quality, realistic picture database. Behavior Research Methods, 46, 596-610.

Masterson, J., Druks, J., \& Gallienne, D. (2008). Object and action picture naming in three- and five-year-old children. Journal of Child Language, 35, 373-402.

Matsumoto, D., \& Ekman, P. (1988). Japanese and Caucasian facial expressions of emotion (JACFEE) and neutral faces (JACNeuF) [Slides]. San Francisco: Author.

Mendez, M. F., Lauterbach, E. C., \& Sampson, S. M. (2008). An evidencebased review of the psychopathology of frontotemporal dementia: A report of the ANPA Committee on Research. The Journal of Neuropsychiatry and Clinical Neurosciences, 20, 130-149.

Mikels, J. A., Fredrickson, B. L., Larkin, G. R., Lindberg, C. M., Maglio, S. J., \& Reuter-Lorenz, P. A. (2005). Emotional category data on images from the International Affective Picture System. Behavior Research Methods, 37, 626-630.

Montagne, B., Schutters, S., Westenberg, H. G., van Honk, J., Kessels, R. P., \& de Haan, E. H. (2006). Reduced sensitivity in the recognition of anger and disgust in social anxiety disorder. Cognitive Neuropsychiatry, 11, 389-401.

Murphy, N. A., \& Isaacowitz, D. M. (2008). Preferences for emotional information in older and younger adults: A meta-analysis of memory and attention tasks. Psychological Aging, 23, 263-286.
Ochsner, K. N. (2008). The social-emotional processing stream: Five core constructs and their translational potential for schizophrenia and beyond. Biological Psychiatry, 64, 48-61.

Olofsson, J. K., Nordin, S., Sequeira, H., \& Polich, J. (2008). Affective picture processing: An integrative review of ERP findings. Biological Psychology, 77, 247-265.

Olsson, A., \& Ochsner, K. N. (2008). The role of social cognition in emotion. Trends in Cognitive Sciences, 12, 65-71.

Orobio de Castro, B., Merk, W., Koops, W., Veerman, J. W., \& Bosch. (2005). Emotions in social information processing and their relations with reactive and proactive aggression in referred aggressive boys. Journal of Clinical Child and Adolescent Psychology, 34, 105-116.

Riegel, M., Zurawski, L., Wierzba, M., Moslehi, A., Klocek, L., Horvat, M., ... \& Marchewka, A. (2016). Characterization of the Nencki Affective Picture System by discrete emotional categories (NAPS BE). Behavior Research Methods, 48, 600-612.

Ryan, R. M., \& Deci, E. L. (2000). Intrinsic and extrinsic motivatons: Classic definitions and new directions. Contemporary Educational Psychology, 25, 54-67.

Schock, J., Cortese, M. J., \& Khanna, M. M. (2012). Imageability estimates for 3,000 disyllabic words. Behavior Research Methods, 44, 374-379.

Sedikides, C., Olsen, N., \& Reis, H. T. (1993). Relationships as natural categories. Journal of Personality and Social Psychology, 64, 7182.

Snodgrass, J. G., \& Vanderwart, M. (1980). A standardized set of 260 pictures: Norms for name agreement, image agreement, familiarity, and visual complexity. Journal of Experimental Psychology: Human Learning and Memory, 6, 174-215.

Székely, A., \& Bates, E. (2000). Objective visual complexity as a variable in studies of picture naming. Center for Research in Language Newsletter, 12, 3-33.

Tottenham, N., Tanaka, J. W., Leon, A. C., McCarry, T., Nurse, M., Hare, T. A., ... \& Nelson, C. (2009). The NimStim set of facial expressions: Judgments from untrained research participants. Psychiatry Research, 168, 242-249.

Tukey, J. W. (1977). Exploratory data analysis. Reading: Addison-Wesley.

Uekermann, J., Kraemer, M., Abdel-Hamid, M., Schimmelmann, B. G., Hebebrand, J., Daum, I., ... \& Kis, B. (2010). Social cognition in attention-deficit hyperactivity disorder (ADHD). Neuroscience and Biobehavioral Reviews, 34, 734-743.

Uljarevic, M., \& Hamilton, A. (2013). Recognition of emotions in autism: A formal meta-analysis. Journal of Autism and Developmental Disorders, 43, 1517-1526.

Warriner, A. B., Kuperman, V., \& Brysbaert, M. (2013). Norms of valence, arousal and dominance for 13,915 English lemmas. Behavior Research Methods, 45, 1191-1207.

Willis, J., \& Todorov, A. (2006). First impressions: Making up your mind after a $100-\mathrm{ms}$ exposure to a face. Psychological Science, 17, $592-598$. 